\title{
Turning On a Light Beam with a Single Molecule
}

\author{
A single molecule can switch a beam of photons on or off, a potentially \\ useful function for a quantum computer.
}

By Katherine Wright

$\mathrm{R}$ esearchers have demonstrated that a single molecule can switch a light beam on or off, a function that could be useful for controlling elements of a quantum computer or a network of such computers [1]. In demonstrating the switching, the team observed a quantum effect called vacuum Rabi splitting, which has not previously been observed in single molecules. The system used by the team, which consists of a molecule coupled to an optical cavity, could also act as a quantum bit, or qubit, adding to the list of qubit candidates.

When considering potential components for quantum technologies, researchers want "as many options as possible," says Vahid Sandoghdar of the Max Planck Institute for the

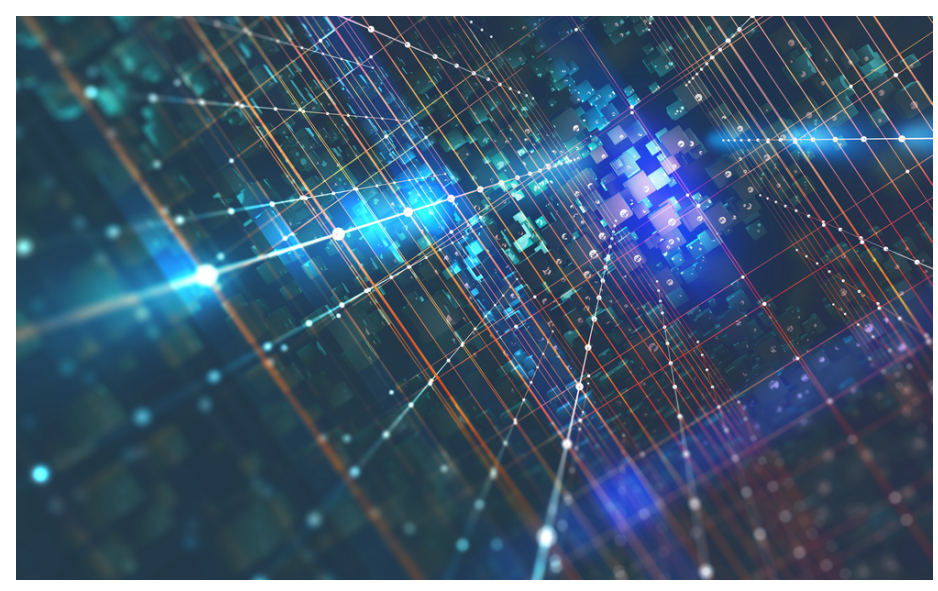

Quantum tech tools. Experiments show that single molecules have superior properties for controlling light, making them potential building blocks for future quantum technologies. Credit: Siarhei/stock.adobe.com
Science of Light, Germany. He and his colleagues have been exploring single molecules, which have been overlooked by most others in the field.

Molecules are small and easy to fabricate, two beneficial properties for quantum technologies. Sandoghdar and his colleagues have ideas for how they could be used as qubits, but they have also found that molecules have superior properties for another use: controlling light.

To study the interactions of a single molecule with light, the team placed an ultrathin crystal containing a small number of the molecules of interest-a dye called dibenzoterrylene (DBT)-inside an optical cavity formed between a flat mirror and a curved mirror. They cooled the whole experiment to $4 \mathrm{~K}$ and adjusted the cavity so that its resonant frequency matched the frequency of the photons the molecule absorbs and emits as it makes transitions between its ground state and its main excited state. The team then illuminated the cavity containing DBT with a laser and recorded the transmitted light.

The resulting transmission spectra display two clear peaks, an effect known as vacuum Rabi splitting. This effect is seen in other quantum systems but had not been observed for a single molecule. The team's data show that the Rabi splitting comes from an exchange of photons between the molecule and the cavity and indicate so-called strong coupling of the molecule to light. This coupling is key for quantum processors, for example, where researchers envision transferring data stored in matter into light signals.

The team's data also reveal that the light-matter interactions of the system are highly nonlinear. For example, as the power of 
the input laser was increased, the transmission of the molecule-cavity system increased up to a point and then stopped increasing, a classic sign of nonlinearity. In this case, the maximizing, or "saturation," of the output light was reached with very little input light. Even a single photon every 100 picoseconds or so-the natural timescale for this cavity (the cavity "lifetime")-was enough to get close to saturation. This nonlinearity is important for switches, Sandoghdar says, because it means that the molecule's transmission of light can be dramatically changed with only a small change in the input light.

The team used this nonlinearity in a final set of experiments in which they illuminated the molecule-cavity system with two lasers with slightly different frequencies. They set up the conditions so that the first laser, on its own, was completely blocked by the molecule. The researchers then turned on the transmission of this laser by illuminating with the second laser, which was tuned to have such a strong interaction with the molecule-cavity system that its photons fully occupied it. The first laser beam could then pass through as if the molecule and cavity weren't there. Analysis of the process showed that the switch to a transmitting state was induced by a single photon per cavity lifetime. This feat normally requires macroscopic blocks of a material that contain a huge number of atoms or molecules, Sandoghdar says. "Here we show that we can do it with only one."

"It has long been a dream to leverage [the] properties of single-molecule systems for real-world applications," says Josh Hihath, a computer and electrical engineer at the University of California, Davis, whose research focuses on understanding the physical properties of molecular-scale systems. The demonstrations by Sandoghdar and his colleagues "will open new avenues for applications [of molecules] in fields including quantum sensing, [quantum] computation, and photonics."

Katherine Wright is the Deputy Editor of Physics.

\section{REFERENCES}

1. A. Pscherer et al., "Single-molecule vacuum Rabi splitting: Four-wave mixing and optical switching at the single-photon level," Phys. Rev. Lett. 127, 133603 (2021). 